\title{
How Football Players Determine where to Run to Tackle other Players: A Mathematical and Psychological Description and Analysis
}

\author{
Dennis M. Shaffer ${ }^{1, *}$ and Thomas B. Gregory ${ }^{2}$ \\ ${ }^{I}$ Department of Psychology, The Ohio State University-Mansfield, ${ }^{2}$ Department of Mathematics, The Ohio State \\ University-Mansfield, USA
}

\begin{abstract}
Players in American football must be able to competently and quickly intercept and tackle an opponent who has the ball. We developed a mathematical model that describes the use of a constant target-heading angle between the pursuer and ball carrier. We found that players in American football maintained a constant target-heading angle across changes in initial angle, direction, and speed of the players being pursued. Players more often evaded capture by running toward the chaser and then changing direction, than by running faster and farther away from the chaser. Our mathematical model made several testable predictions that were supported by our data. It also makes several testable predictions that may be investigated in future work. The act of maintaining a constant target-heading angle seems to be a strategy that is used generically to pursue and intercept moving objects.
\end{abstract}

\section{INTRODUCTION}

Defensive players in American football must be able to know precisely the angle and speed at which to run in order to competently and quickly intercept and tackle an opponent who has the ball. With little training, players are remarkably good at chasing and catching ball carriers in an open field, even when the ball carrier changes direction and/or speed while running. Predators pursuing prey, humans walking or driving through cluttered environments while trying to avoid collisions, and military personnel attempting to intercept ballistic targets with missiles, face similar navigational challenges. In the present work, we examine three strategies which pursuing players use in American football. Each of these strategies is based on the information presented in Fig. (1). We denote $\beta$ as the angle between the pursuer's instantaneous direction of locomotion and the line segment connecting the pursuer to the ball carrier. If the pursuer's direction of motion is directly toward the ball carrier, $\beta$ will be zero; if it is ahead of the ball carrier it will be positive; if behind, it will be negative.

The first potential strategy is called pursuit. A diagram of the pursuit strategy is shown in the left panel of Fig. (2). This strategy is also called 'aiming,' 'homing' or 'centering,' because the pursuing player's objective is to aim directly toward the ball carrier (i.e., to center his direction of motion on the ball carrier). In this case, the pursuer aims directly toward the ball carrier at each instance in time, so that $\beta=0$ at all times. Some theoretical models using differential equations to determine best strategies in 2- and 3-dimensional space have supported the use of the pursuit strategy [1-3]. There is some behavioral evidence for the use of this strategy in nature from studies of houseflies chasing one another [4], teleost fish chasing food from above [5], and humans

*Address correspondence to this author at the Department of Psychology, The Ohio State University, Mansfield, USA; E-mail: shaffer.247@ osu.edu walking to stationary or slowly moving targets in an open field $[6,7]$. Young children running through a circle trying to 'tag' another person (usually unsuccessfully) while playing the game of tag also typically use this strategy [8,9]. As with virtually all other strategies, this strategy is only effective as long as the pursuer's speed is great enough so that the pursuer can overtake the ball carrier.

A second, less likely alternative is the classic predictive strategy. In this strategy, the pursuer runs in a straight line to a spot the ball carrier will go and waits until the ball carrier gets there to tackle him without having to look at the ball carrier once they started running. Archer fish catching their dislodged prey use this strategy $[10,11]$. This requires a ballistic trajectory that will not change course due to the pursuee (e.g., a dead insect). In the case of a ball carrier, this would be the case if the pursuer knew exactly where the ball carrier was going to run along a ballistic path and ran to that spot and waited without having to use instantaneous direction of locomotion. In times past, mariners used a similar strategy, called 'dead reckoning'; it was their only way to map a course when the sky was overcast and a celestial observation was not possible.

Another strategy is to maintain a constant bearing angle (or CBA). There is behavioral evidence for two different methods of employing this strategy. First, boaters and airplane pilots are taught that if another craft maintains a constant bearing angle relative to them as the distance between them diminishes, and there is no change in course or speed over time, they are on a collision course and evasive action is called for [12]. Maintaining the target's bearing direction here requires that there is a visible external reference frame such as a distant landmark [13]. Behavioral evidence that supports this comes from dragonflies intercepting prey overhead by maintaining a constant angle between the target and horizon [14]. We refer to this method of employing the strategy throughout the manuscript as CBA. 

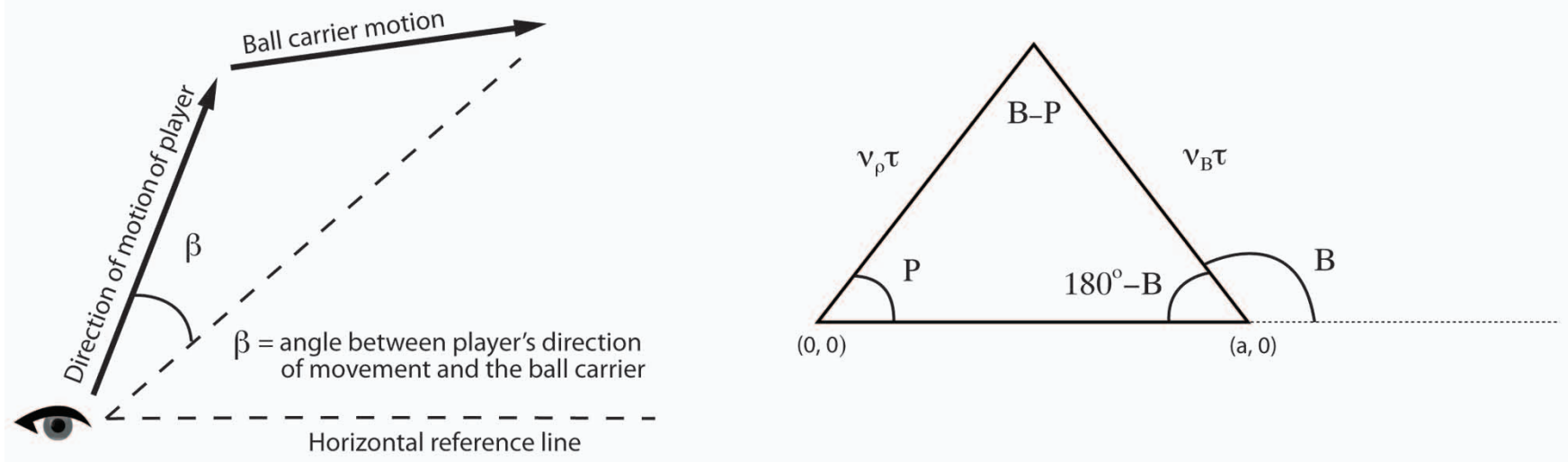

Fig. (1). Layout for moving to a ball carrier. $\beta$ is the angle between the player's direction of movement and the ball carrier relative to a constant reference line. If the player's direction of movement is lined up with the ball carrier, $\beta$ will be zero.

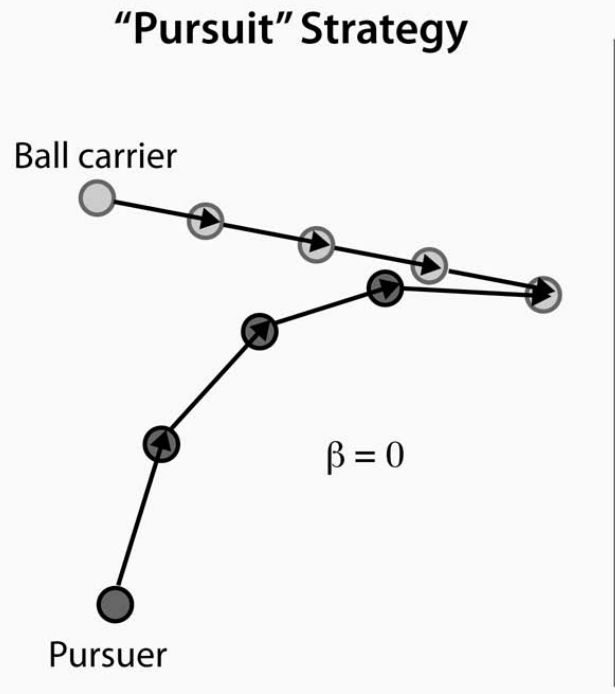

\section{“Interception" Strategy}

Ball carrier

Fig. (2). (left panel) Layout of the pursuit strategy. During pursuit, the pursuer's objective is to aim directly toward the ball carrier at each instant in time, nulling $\beta$. (right panel) Layout of the constant target-heading-angle strategy. The objective of the pursuer is to aim slightly ahead of the ball carrier, nulling $\mathrm{d} \beta / \mathrm{dt}$ while keeping $\beta$, or the angle itself, greater than zero.

The second method of employing this strategy is to move in such a way so that the target-heading angle remains constant [13]. A diagram of this strategy is shown in the right panel of Fig. (2). This strategy is also known as 'predictive pursuit' or 'interception.' The goals of this strategy are, first, to aim slightly ahead of where the ball carrier is going, so that $\beta>0$ at all times, and, second, to maintain a constant angle; i.e., $\beta_{l}=\beta_{2}=\beta_{n}$, so that the derivative of the angle with respect to time is equal to zero (i.e., $\mathrm{d} \beta / \mathrm{d} t=0$ ). This strategy produces a collision course as long as $\beta$ is less than ninety degrees and the pursuer moves in approximately a straight line [15] or turns onto a straight interception path [13]. We refer to this method of employing the strategy throughout the manuscript as CTHA. Theoretical models using differential calculus and graphical analysis to describe best strategies for automatic target-seeking devices, and for launching torpedoes at ships, demonstrate the superiority of using a CTHA strategy over a pursuit or homing strategy [8,
16]. Also, for some types of situations of football players pursuing other football players, some theoretical tests have shown that a similar (to CTHA) strategy, the so-called 'proportional leading' or 'mirroring' strategy, in which the pursuer mirrors the path of the ball carrier to achieve interception, is most successful $[9,17]$. Behavioral evidence for the CTHA strategy comes from male hoverflies (Eristalis) chasing projectiles [18], hoverflies (Syritta pipiens L.) during circling and sideways tracking maneuvers, teleost fish chasing food from below [5], and Big Brown bats (Eptesicus fuscus) echolocating toward prey [19]. An abundance of evidence from humans interacting with targets shows the use of the CTHA strategy. For instance, peoples' judgments about whether they will collide with a moving target are correlated with the rate of change in the target-heading angle [20]. People intercepting targets while riding on a tricycle [21], walking on a treadmill [22], or walking or controlling their velocity via a joystick in a virtual environment $[15,23,24]$ 
all use a CTHA strategy. Additionally, older children (10-12year-olds) adhere more to a CTHA than younger children (57-year-olds) [25]. It is due to the wealth of evidence for the use of CTHA by humans intercepting faster-moving targets that we hypothesized that football players pursuing other football players would also use this strategy.

To summarize, the pursuit strategy predicts that pursuers will aim directly at ball carriers as they run to catch them. This will result in a curved running path and a target-heading angle $\beta$ equal to zero. The CBA strategy can only be used with a fixed external reference frame, like a fixed background or distant landmark, which we did not use in this study, and which is not typically available to players in this situation. The CTHA strategy predicts that the pursuers will move in straight-line paths, the target-heading angle $\beta$ will be greater than zero, and the change in the target-heading angle with respect to time will be equal to zero (i.e., $\mathrm{d} \beta / \mathrm{d} t=0$ ). The classic predictive strategy predicts that pursuers will run to the interception point and wait there to intercept the ball carrier. In the following several paragraphs we develop a mathematical model outlining the constant target-heading angle strategy.

\section{MATHEMATICAL MODEL OF THE CONSTANT TARGET-HEADING ANGLE STRATEGY}

The right panel of Fig. (1) shows a Cartesian-coordinate system that has as its origin $(0,0)$ the starting point of the pursuer, and has as the positive ray of its $x$-axis the line connecting the pursuer to the ball carrier. The angle that the pursuer's path makes with the positive $x$-axis is designated as $P$, and the angle that the ball carrier's path makes with the positive $x$-axis is designated as $B$. Thus, the initial reference line between the pursuer and the ball carrier makes an angle $P$ with the pursuer's path, and an angle $B$ with the ball carrier's path. Since we are measuring both $P$ and $B$ in the counterclockwise direction, from the part of the $x$-axis to the right of each football player, the players will be running in parallel directions when the $P$ and $B$ are equal. Also, at the instant at which the ball carrier and his pursuer start to run, $P$ and $\beta$ are equal, as they are when either player changes direction, and the problem reinitializes.

We will denote the distance between the pursuer and the ball carrier, at $t$ seconds after they begin to run, or $t$ seconds after the problem has reinitialized, by $a(t)$; consequently, the ball carrier begins running from the point $(a(0), 0)$ in the right panel of Fig. (1). Set $a=a(0)$.

We use the symbols $v_{P}$ and $v_{B}$ to represent the speed of the pursuer and the speed of the ball carrier, respectively, and we let $t_{i}$ stand for the time it takes to intercept. Looking at the right panel of Fig. (1), we conclude from the Law of Sines that

$\sin (P)=v_{B} \sin (B) / v_{P}$

so that the greater the angle $P$, the lower the pursuer's speed needs to be, assuming that $B$ and $v_{B}$ remain constant. Also, note that for the angles $P$ that we are considering, $\sin (P)$ increases as $P$ increases. Thus, a pursuer can intercept the ball carrier using the target-heading-angle strategy earlier by increasing speed and making angle $P$ smaller. On the other hand, a slower pursuer might still be able to achieve interception by making angle $P$ greater, assuming the ball carrier were to continue to run in the same straight line.

Since we have denoted the speed of the pursuer by $v_{P}$ and the speed of the ball carrier by $v_{B}$, it follows that after time $t$ the ball carrier $B$ will be at the point

$$
\left(a+t v_{B} \cos (B), t v_{B} \sin (B)\right)
$$

and the pursuer $P$ will be at the point

$$
\left(t v_{P} \cos (P), t v_{P} \sin (P)\right)
$$

The distance between them will then be the square root of

$$
\left(t v_{P} \cos (P)-a-t v_{B} \cos (B)\right)^{2}+\left(t v_{P} \sin (P)-t v_{B} \sin (B)\right)^{2}
$$

Simplifying, we can see that the square of the distance between them will be

$$
\begin{aligned}
& t^{2}\left(v_{P}^{2}+v_{B}^{2}\right)+a^{2}-2 t^{2} v_{P} v_{B} \\
& (\cos (P-B))+2 t a\left(v_{B} \cos (B)-v_{P} \cos (P)\right)
\end{aligned}
$$

The rate at which the square of the distance is changing is the derivative of this expression with respect to $t$; this derivative equals

$$
\begin{aligned}
& 2 t\left(\left(v_{P}^{2}+v_{B}^{2}\right)-4 t v_{B} v_{P}(\cos (P-B))-2 a\right. \\
& \left(v_{P} \cos (P)-v_{B} \cos (B)\right)
\end{aligned}
$$

We are assuming that $B, v_{B}$, and $v_{P}$ are constant with respect to time $t$. Of course, it is in the interest of the pursuer to maximize the rate at which the distance is decreasing. At what angle should the pursuer run so that he can most efficiently diminish the distance between himself and the ball carrier? To find this angle, we must calculate the angle the pursuer should now make with the positive $x$-axis, that is to say, with the line between the pursuer and the ball carrier, in order to make the rate of change of the distance between him and the ball carrier as negative as possible. To find this optimal angle, i.e., value of $P$, we will take the derivative of (6) above with respect to $P$, and set it equal to zero. The derivative with respect to $P$ is

$$
4 t v_{P} v_{B}(\sin (P-B))+2 a\left(v_{P} \sin (P)\right)
$$

When we set this latter expression equal to zero, we can divide by 2 and $v_{P}$. After some mathematical manipulation, we get that

$$
\tan (P)=\left(t v_{B} \sin (B)\right) /\left(\mathrm{t}_{B} \cos (B)+(a / 2)\right)
$$

If we let $d\left(=t v_{B}\right)$ denote the distance run by the ball carrier during time $t$, this latter expression can be simplified to

$$
\tan (P)=(\sin (B)) /(\cos (B)+(a /(2 d)))
$$

Since $\mathrm{a} /(2 \mathrm{~d})$ is never quite zero, the model shows that $P$ should be less than $B$. We noted above that when $P$ is positive, as it is here, the pursuer is aiming ahead of the ball carrier.

Also, we see from (8) that when $t$ is zero, $P$ is zero, as well, so that if the pursuer wants the gap between himself and the ball carrier to be closing as fast as possible immediately, as he might when they are both beginning to run, the pursuer should run toward the ball carrier. As we noted above in analyzing the pursuit strategy, aiming directly at the 
ball carrier results in a curved pursuer path. As $t$ gets bigger, we see from (9) that $a /(2 d)$ becomes less and less important, so that the pursuer's path should more nearly approach a straight line parallel to the path of the ball carrier. Thus, if the pursuer wants the gap between himself and the ball carrier to be decreasing maximally at a time $t$ in the future, like when he was about to tackle, the pursuer should run in more of a straight line, a line more nearly parallel to the path of the ball carrier. Since the bearing will not be changing much, the derivative of $P(\sim \beta)$ with respect to $t$ should be close to zero.

To summarize, our mathematical model, consistent with the CTHA strategy, predicts that the pursuers will move in, or move onto straight-line paths ( $c f$. [13]), the target-heading angle $\beta$ will be greater than zero after the initial steps onto the pursuit path, the change in the target-heading angle with respect to time will be close to zero (i.e., $\mathrm{d} / \mathrm{dt} \sim 0$ ), and when there is a mid-pursuit change of the ball carrier, the change in the target-heading angle after the change will also be equal to zero (i.e., $\mathrm{d} \beta / \mathrm{dt} \sim 0$ ), even though the targetheading angle may change after the mid-pursuit change of the ball carrier.

All previous experiments examining the strategies used to pursue targets have either been done naturalistically or by using targets with ballistic paths. We investigated which of the strategies a pursuer in football uses when chasing a ball carrier, and whether the strategy used during constant speed and constant direction trials is also used when the ball carriers change direction and/or speed midway through their run.

\section{METHOD}

\section{Participants}

Five senior-high-school varsity-football starters participated in the experiment. All players were eighteen, and started at various defensive positions (3 cornerbacks, 1 strong safety and 1 defensive end). All five had played competitive football since they were in junior-high school.

\section{Materials}

A portion of a high school football field $(22.87 \mathrm{~m}$ laterally and $22.87 \mathrm{~m}$ in depth) was used for the pursuit area. This area was first equally divided and outlined by cones placed $4.57 \mathrm{~m}$ apart laterally and in-depth. Smaller cones were placed at $0.91 \mathrm{~m}$ intervals to further divide the field into $0.91 \mathrm{~m} \mathrm{x}$ $0.91 \mathrm{~m}$ units. The ball carrier started at a position that we indicate by the $(0,0)$ coordinate. The pursuer started directly to the right of, and $18.3 \mathrm{~m}$ from the ball carrier. A straight line from the starting positions of the ball carrier and pursuer formed the reference line from which angles at which ball carriers ran were established. Starting from the cone placed at the $(0,0)$ coordinate, the angles were established using a geometric protractor. The "ball carriers" were instructed to run along one of four long strands of yellow yarn that were tied to the $(0,0)$ cone. Each piece of yarn was placed along each of the initial angles $\left(20^{\circ}, 40^{\circ}, 60^{\circ}, 80^{\circ}\right.$, relative to the horizontal reference line). Another ground marker identified the midway point for each running angle, so that the target knew when to change speed and/or direction (if instructed to do so). Field markers were placed behind the four cones specifying the angles to run along so the lines could be seen on the video recording. A camcorder was placed at a distance of $30 \mathrm{~m}$ behind and above the reference line formed by the starting positions of the ball carrier and pursuer. It was positioned behind and above the corner of the grid where the ball carriers began running. We recorded the moment-by-moment positions (in $5 / 30^{\text {th }}$ of a second frames) of both the pursuer and the target.

\section{Design and Procedure}

For a total of 96 trials, one pursuer at a time chased and tried to touch the ball carrier. The 96 randomized trials were split equally into the four initial angle straight-line paths that ball carriers were instructed to run along, specified as $20^{\circ}$, $40^{\circ}, 60^{\circ}$, and $80^{\circ}$, from their starting position $(0,0)$. In addition, for each initial angle, the runner ran in one of the following ways: (1) constant speed /straight line, (2) constant speed /cut up, (3) constant speed /cut down, (4) speed up /straight line, (5) speed up/cut up, and (6) speed up /cut down. In the 'cut' condition, runners began running along a straight line along one of the angle paths from their initial starting position, and midway changed the angle they were running by $+/-20$ degrees. For instance, if the runner was instructed to begin running along the 20 degree path and cutup to the 40 degree angle, he would cut midway on the 20 degree path and begin running at the cone specifying 40 degrees. In the 'cut down' condition, the runner in the previous example would change midway to running in a straight line to 0 degrees. In the speed up condition, runners were told to run faster after the midway point than they were running before the midway point.

Before each trial, one football player was chosen to be the ball carrier for that particular trial, and was given specific instructions on what speed and angle he must take, and whether and in which direction he would cut. Two targets started running along with the ball carrier, but stopped running after $\sim 3 \mathrm{~m}$. This was done in order to eliminate any cognitive expectations concerning characteristics of the ball carrier or running path that might influence the central strategy that the pursuers use. The pursuer started $18.3 \mathrm{~m}$ directly to the right of the starting position of the target and runners, and was instructed to touch the runner before he ran past the cones marking the end line for the running area. Because there was a considerable amount of running involved, each of the five football players received between five and ten trials of being the ball carrier and between five and ten trials being the pursuer. While pursuers could see the markings on the field, just as the ball carriers could, they would have to choose the correct running path and speed from a set of 24 possible combinations of angle, speed, and cut. In addition, we saw no obvious signs from any of the pursuers that they could correctly anticipate the running path and speed of the ball carriers.

Due to perspective foreshortening, the area of the field formed a trapezoid. Lines were drawn on a transparency placed over the television screen from one cone in depth to its opposite counterpart on the other side of the field (this was done with cones both in-depth and laterally). Each of the $0.91 \mathrm{~m}$ by $0.91 \mathrm{~m}$ grids was then further divided into $3 \times 3$ unit grids. This was done in order to transform the trapezoidal coordinates into Euclidean coordinates. The pursuer and ball carrier's foot positions were then marked at each $5 / 30^{\text {th's }}$ of a second (or every $0.167 \mathrm{~s}$ ). These foot positions then would have a potential error equivalent to $0.3 \mathrm{~m}$ in-depth and $0.3 \mathrm{~m}$ laterally, or, given the section discussing speed below, 
an error of approximately $0.3 \mathrm{~m} \times 0.3 \mathrm{~m}$ for every $0.95 \mathrm{~m}$ moved (average speed of the pursuer and ball carrier for every 5 frames).

\section{RESULTS}

Eighty-seven trials in which the pursuer and ball carrier remained visible in the video were coded. This data was pooled across participants for all of the following analyses, except for one in which we directly compared the change in target-heading angle across participants. Seventy-seven out of eighty-seven $(\sim 89 \%)$ ended in successful interceptions. There was $100 \%$ agreement between 2 independent raters that pursuers were using a classic predictive strategy in only three codable trials (i.e., Cronbach's $\alpha=1$ ). These were separated from the remaining 74 trials, because by definition, pursuers cannot be using either a pursuit or interception strategy if they are already using a classic predictive strategy. In a classic predictive strategy, pursuer would know ahead of time exactly where the ball carrier was going on a ballistic path and stop and wait for them to get there, without having to use instantaneous direction of locomotion.

Correlational analyses were performed on the entire path ( $\mathrm{x}$ and $\mathrm{z}$, lateral and depth positions at each moment in time) for the trials in which the ball carrier ran in a straight line, and for the two separate paths (before-after) for the 'cut' conditions. Here we report Pearson correlation values for the straight condition, both cut conditions, and all of the conditions. The sample of $r$-values was highly negatively skewed for every condition (skewness $(S K)=-2.55$, standard error
$(S E)=0.44, S K=-2.73, S E=0.25$, and $S K=-2.65, S E=$ 0.22 , for the straight condition, both cut conditions, and all of the conditions, respectively). Therefore, we report median $r$-values as well as mean $r$-values. The skewness is expected, given that in the cut down condition from $20^{\circ}$ to $0^{\circ}$ the pursuer started running one way in depth and then had to turn around and run the opposite way before catching the ball carrier. The median $r$-value for the straight condition, both cut conditions, and all of the conditions was 0.97 , mean $(M)=0.9$, standard deviation $(S D)=0.18$ (they were the same for every category). A paired-samples t-test showed that there was no difference in r-values in the cut conditions for paths prior to the cut compared to those after the cut, $t(45)=1.57, p>.01$. This means that pursuers ran in remarkably straight lines irrespective of condition. A straight line accounted for a median of $94 \%$ of the variance in running paths for all trials, $M=84 \%, S D=0.24$. While our data do show that pursuers ran very close to straight lines, it would be difficult to say whether they ran in straight lines the entire way, or whether they turned slightly onto a straight-line interception path [13]. This is because we did not begin coding the pursuer's position until the ball carrier ran past the other two players who ran beside him for the first $3 \mathrm{~m}$ as stated in the Method section. The mean targetheading angle, $\beta$, was $50.11^{\circ}\left(\right.$ range $\left.=29^{\circ}-77^{\circ}, \mathrm{SD}=9.86^{\circ}\right)$; this result supported the conclusion that pursuers were using a CTHA strategy, rather than a pursuit strategy, by aiming ahead of their targets and not aiming directly at them as they ran to catch them, $t(73)=43.7, p<.001$. Representative running paths in the various conditions are shown in Fig. (3).

Pursuer \#1, $20^{\circ}-40^{\circ}$, Constant Speed

Pursuer \#3, 40 $0^{\circ}$ Straight, Constant Speed

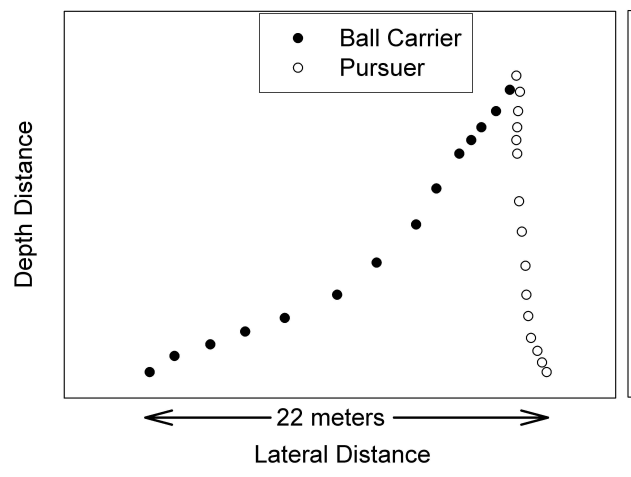

Pursuer \#4, $40^{\circ}-60^{\circ}$, Constant Speed

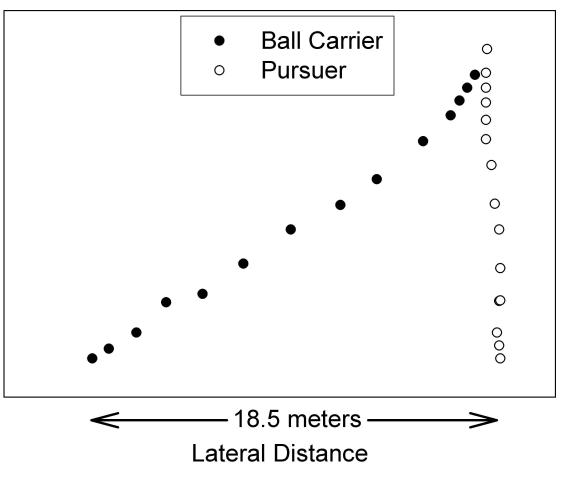

Pursuer \#5, 60-80 , Speed Up

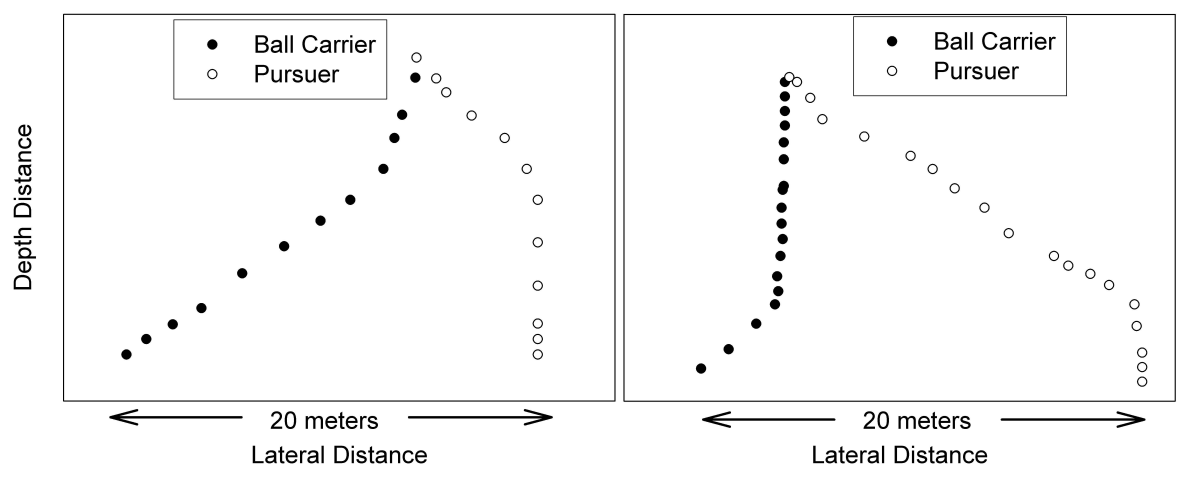

Fig. (3). Representative running paths of pursuers and ball carriers. Four different pursuers are shown chasing ball carriers in 4 different conditions. Pursuers are shown in filled symbols while ball carriers are shown in open symbols at every $5^{\text {th }}$ frame (or at every $0.17 \mathrm{~s}$ ). 
Videos of representative running paths are also shown in the supportive/supplementary 'material. Also consistent with pursuers' use of a CTHA strategy was the finding that the average angular changes for each trial oscillated around 0 , with a mean $\mathrm{d} \beta / \mathrm{dt}$ value of $1.66 \% 17 \mathrm{~s}(S D=1.28)$. Among all conditions, the change in target-heading angle ranged from $1.51 \% .17 \mathrm{~s}-1.77 \% .17 \mathrm{~s}$. Average angular changes for each subject, initial angle, cut and speed condition are shown in Table 1. As Table 1 shows, overall average angular change was identical in the subject, cut, and speed condition $\left(1.66^{\circ} \% 17 \mathrm{~s}\right)$ and virtually identical in the initial angle position $(1.39 \% .17 \mathrm{~s})$. We first analyzed whether there were differences in $\mathrm{d} \beta / \mathrm{dt}$ across subject, cut, speed, and initial angle conditions. Four separate analyses of variance (ANOVAs) indicated that the change in target-heading angle, $\mathrm{d} \beta / \mathrm{dt}$, was the same irrespective of pursuer, $F(4,73)=0.32, p>.1$, change in direction, $F(2,74)=0.06, p>.1$, change in speed, $F(1,74)=1.23, p>.1$, and initial starting position, $F(3,73)=$ $2.17, p=.1$. Since no differences in $\mathrm{d} \beta / \mathrm{dt}$ were found across subjects, cuts, speeds, and initial angles, we collapsed the conditions to analyze whether $\mathrm{d} \beta / \mathrm{dt}$ was significantly different from zero across all conditions (and trials). A one-sample t-test comparing the average $d \beta / d t$ across all trials to zero indicated that the mean angular change was not significantly different from $0(t(810)=0.54, p>.5)$. We further divided the data in the 'cut' conditions and evaluated whether the changes in target-heading angle before the cut were different from changes after. For the 43 'cut' conditions, we performed separate ANOVA's and found that there were no differences before and after the cut in the way that changes in the target-heading angle maintained, all $p$ 's $>.25$. Interestingly, in $40 \%$ of the trials, the target-heading angle itself changed significantly after the cut. Thus, though the pursuer may take a different angle, the pursuer still attempted to maintain the change in the new target-heading angle around $0 \% 17 \mathrm{~s}$. Plots of changes in target-heading angle over time corresponding to the running path trials shown in Fig. (3) are shown in the top and bottom panels of Fig. (4).
Speed of Targets and Pursuers in Constant Speed and Speed-up Conditions

In order to make sure that targets were running at a relatively constant speed during the constant-speed trials and were speeding up during the speed-up trials, we measured the average speed of both players for the constant-speed and speed-up conditions by measuring the distance chased per number of video frames, and converted this into meters per second. The means of the before- and after-portions for the ball carrier in the constant-speed trials were not significantly different $\left(5.82 \mathrm{~ms}^{-1}\right.$ and $5.53 \mathrm{~ms}^{-1}$ for the pursuee and pursuer, respectively). The average speed of the ball carrier and pursuer before and after the midway point for speed-up conditions were: Ball carrier: $5 \mathrm{~ms}^{-1}$ (before), $6.92 \mathrm{~ms}^{-1}$ (after), and Pursuer: $3.73 \mathrm{~ms}^{-1}$ (before) and $7.45 \mathrm{~ms}^{-1}$ (after).

\section{Unsuccessful Interceptions}

We also analyzed unsuccessful interceptions. Of the 13 trials where the target was not caught, 2 occurred when the target was running the farthest away from the pursuer and in the condition where the target sped up midway through the pursuit. Nine out of the thirteen trials were in a condition where the target was running directly at the pursuer, then changed course in mid-path. Achieving evasion was significantly more effective when running virtually directly toward the pursuer, and then changing direction so as to move in a direction in which the pursuer was not facing, $\chi^{2}(1, N=13)=34.71, p<.001$.

\section{DISCUSSION}

This work supports the premise that players in American football use a constant-target-heading-angle strategy in order to pursue and catch other players. Consistent with this, our work also supports the predictions made by our mathematical model. Players pursue and catch other players by keeping the target-heading angle greater than zero after the initial

Table 1.

\begin{tabular}{|c|c|c|c|}
\hline Subjects & Mean Angular Change & Initial Angles & Mean Angular Change \\
\hline \hline $1(\mathrm{n}=13)$ & $1.68^{\circ}$ & $20^{\circ}(\mathrm{n}=11)$ & $2.06^{\circ}$ \\
\hline $2(\mathrm{n}=14)$ & $1.74^{\circ}$ & $40^{\circ}(\mathrm{n}=20)$ & $1.94^{\circ}$ \\
\hline $3(\mathrm{n}=9)$ & $1.25^{\circ}$ & $80^{\circ}(\mathrm{n}=24)$ & $1.70^{\circ}$ \\
\hline $4(\mathrm{n}=23)$ & $1.80^{\circ}$ & Overall & $1.39^{\circ}$ \\
\hline $5(\mathrm{n}=15)$ & $1.59^{\circ}$ & Cuts & $1.85^{\circ}$ \\
\hline Overall & $1.66^{\circ}$ & Straight $(\mathrm{n}=28)$ & $1.49^{\circ}$ \\
\hline Speed & & Cut up (n=25) & $1.60^{\circ}$ \\
\hline Constant $(\mathrm{n}=39)$ & $1.57^{\circ}$ & Cut down $(\mathrm{n}=21)$ & $1.66^{\circ}$ \\
\hline Speed up (n=35) & $1.76^{\circ}$ & Overall & \\
\hline Overall & $1.66^{\circ}$ & & \\
\hline
\end{tabular}

${ }^{1}$ Please see video 1-6 in the supportive/supplementary material on The Open Sports Sciences Journal website. pursuit and keeping the change in the target-heading angle equal to zero. When faced with a target that changed direction and/or speed, the pursuer established a new target- 
Pursuer \#1, $20^{\circ}-40^{\circ}$, Constant Speed Change in Angle of Pursuit (d $\beta / \mathrm{dt}$ ) Over Time

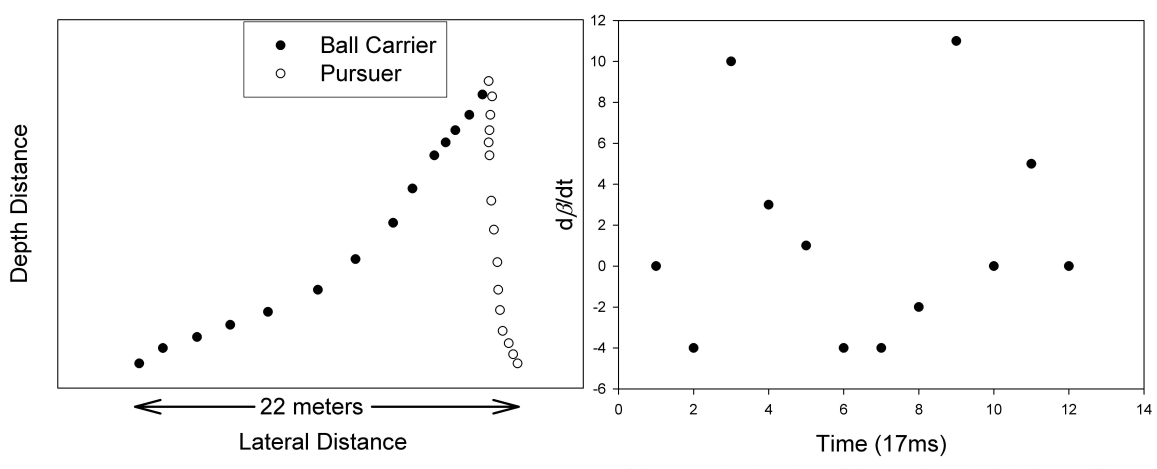

Pursuer\#3, $40^{\circ}$, Straight, Constant Speed Change in Angle of Pursuit (d $\beta / \mathrm{dt}$ ) Over Time

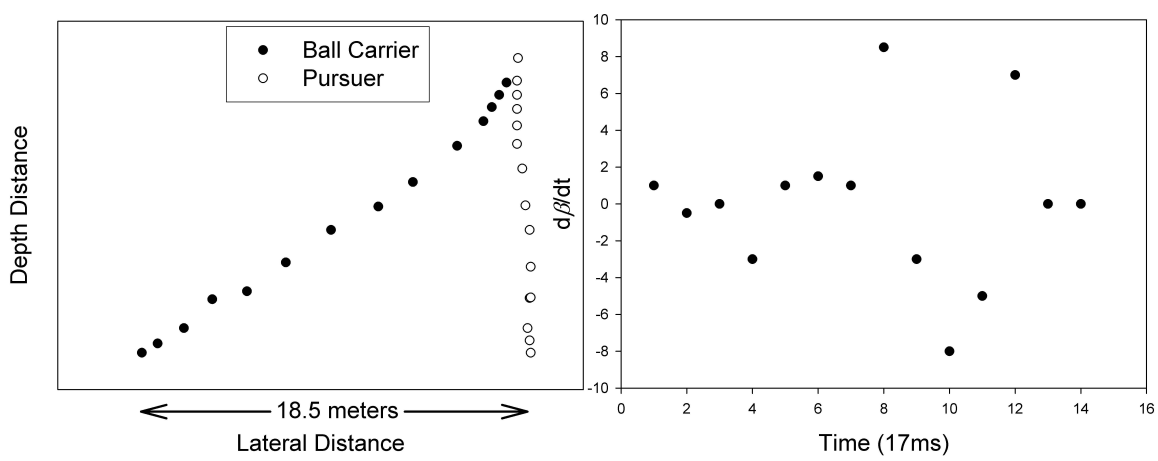

Pursuer \#4, $40^{\circ}-60^{\circ}$, Constant Speed

Change in Angle of Pursuit $(\mathrm{d} / \mathrm{dt})$ Over Time

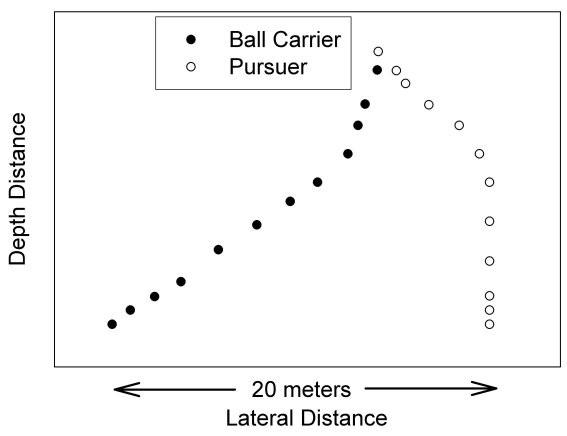

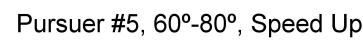

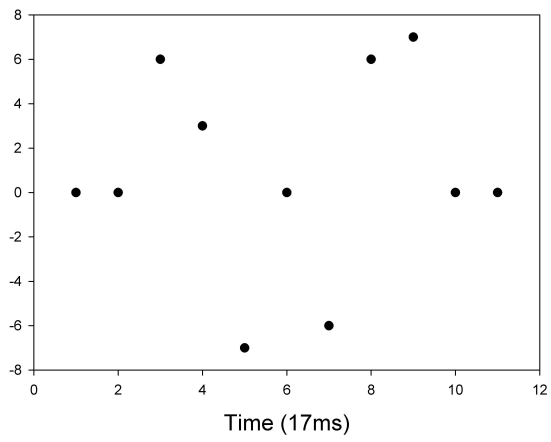

Change in Angle of Pursuit $(\mathrm{d} / \mathrm{dt})$ Over Time
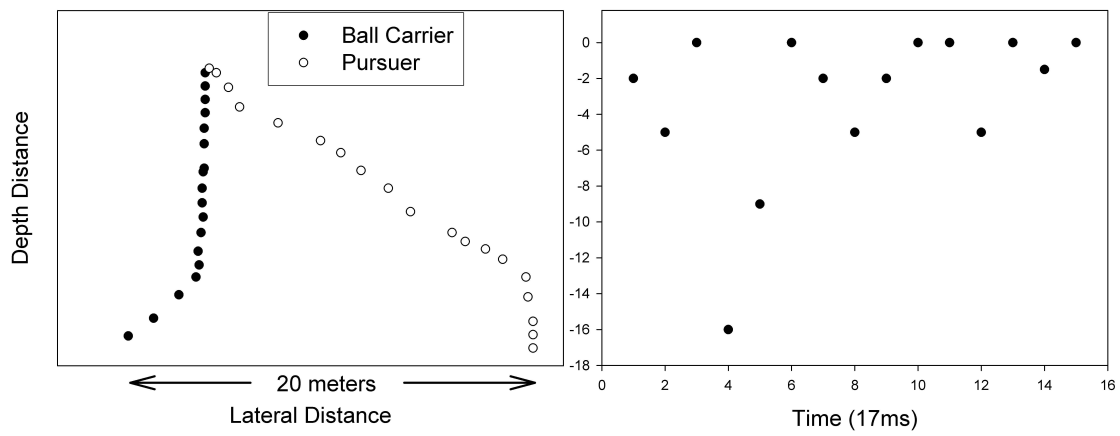

Fig. (4). Changes in the target-heading angle. In both panels, running paths shown in Fig. (3) are shown on the left, while corresponding changes in the target-heading angle are shown to the right.

heading angle, and then maintained the change in the new target-heading angle equal to zero. In fact, as the mathematical model predicts, the CTHA strategy was robust with respect to changes in initial angle, target, changes in direction, and changes in speed.
Evidence for the CTHA strategy also comes from our analysis of unsuccessful interceptions. Most people would probably guess that a player's best bet of not getting caught would be to run faster and in a direction away from a pursuer. However, achieving evasion was significantly 
more effective when using the CTHA strategy against the pursuer-that is, it was more effective for the ball carrier to evade the pursuer by running virtually directly toward the pursuer, and then changing direction so as to move in a direction in which the pursuer was not facing. One of the reasons this strategy is so effective is that a change in targetheading angle is more dramatic when the target is very close to the pursuer. No matter how far and fast a ball carrier runs away from a potential tackler, he cannot drastically change the bearing angle. While the ball carrier may evade pursuit this way by having greater speed and/or endurance than the pursuer, he does not do much to keep the pursuer from knowing where he is headed. This is consistent with a wellknown tactic that ball carriers use to evade defensive players. Typically a ball carrier faces a defensive player and gets him to lean one way, whereupon the ball carrier moves the opposite way. This is also not inconsistent with our mathematical model, which shows that even a slower pursuer might still be able to achieve interception by making angle $P$ greater. Video \#'s 7-8 in the supportive/supplementary material show a representative example of a ball carrier evading pursuit by initially running at $20^{\circ}$, then making a cut to ${ }^{2} 0^{\circ}$.

While we have not specified the available perceptual information that might be used by the pursuer to achieve a CTHA, possible sources include egocentric orientation to the target, the optic flow of information around the eyes and the target, proprioceptive information, and composite tau [15, 27-30].

Future work needs to be done to investigate other characteristics of our mathematical model. First, the model shows that faster pursuers can intercept the ball carrier using the target-heading-angle strategy earlier by increasing speed and making angle $P$ smaller. On the other hand, a slower pursuer might still be able to achieve interception by making angle $P$ greater. It would be interesting to test whether faster pursuers are more likely to intercept ball carriers earlier, and slower pursuers later, and within what window of angles of $P$ interception is most optimal and most likely to occur for pursuers varying in speed. For now, our mathematical model nicely describes how interception takes place in nature, and makes some testable predictions beyond those described by adhering to a CTHA.

\section{SUPPLEMENTARY MATERIAL}

Supplementary material can be viewed at www.bentham.org/open/tocryj

\section{REFERENCES}

[1] Barton JC, Eliezer CJ. On pursuit curves. J Aust Math Soc Ser B 2000; 41: 358-71.

[2] Guha A, Biswas SK. On Leonardo da Vinci's cat and mouse problem. Bull Inst Math Appl 1994; 30: 12-5.

[3] Colman WJA. A curve of pursuit. Bull Inst Math Appl 1991; 27: 45-7.

${ }^{2}$ Please see video 7-8 in the supportive/supplementary material on The Open Sports Sciences Journal website.
[4] Land MF, Collett TS. Chasing behaviours of houseflies (Fannia canicularis). A description and analysis. J Comp Phys 1974; 89: 331-57.

[5] Lanchester BS, Mark RF. Pursuit and prediction in the tracking of moving food by a teleost fish (Acanthaluteres spilomelanurus). J Exp Psychol: Gen 1975; 63: 627-45.

[6] Fajen BR, Warren WH. Behavioral dynamics of steering, obstacle avoidance, and route selection. J Exp Psych: Hum Percept Perform 2003; 29: 343-62.

[7] Rushton SK, Harris JM, Lloyd MR, Wann JP. Guidance of locomotion on foot uses perceived target location rather than optic flow. Curr Biol 1998; 8, 1191-4.

[8] Mungun CE. A classic chase problem solved from a physics perspective. Eur J Phys 2005 ; 26: 985-90.

[9] O'Connell J. Pursuit and evasion strategies in football. Phys Teach 1995; 33: 516-8.

[10] Rossel S, Corlija J, Schuster S. Predicting three-dimensional target motion: How archer fish determine where to catch their dislodged prey. J Exp Biol 2002; 205: 3321-6.

[11] Wohl S, Schuster S. Hunting archer fish match their take-off speed to distance from the future point of catch. J Exp Biol 2006; 209: 141-51.

[12] Pollack HN. Play Ball. Science 1995; 268: 1681.

[13] Fajen BR, Warren WH. Behavioral dynamics of intercepting a moving target. Exp Brain Res 2007; 180: 303-19.

[14] Olberg RM, Worthington AH, Venator KR. Prey pursuit and interception in dragonflies. J Comp Phys A 2000; 186: 155-62.

[15] Fajen BR, Warren WH. Visual guidance of intercepting a moving target on foot. Perception 2004; 33: 689-715.

[16] Yuan LC.-L. Homing and navigation courses of automatic targetseeking devices. J Appl Phys 1948; 19: 1122-8.

[17] Dunbar SR. Chase problems. UMAP J 1994 ; 15: 351-356.

[18] Collett TS, Land MF. Visual control of flight behaviour in the hoverfly, Syritta pipiens L. J Comp Phys 1975; 99: 1-66.

[19] Simmons JA, Dear SP, Ferragamo MJ, Haresign T, Fritz J. Representation of perceptual dimensions of insect prey during terminal pursuit by echolocating bats. Biol Bull 1996; 191: 109-21.

[20] Cutting JE, Vishton PM, Braren PA. How we avoid collisions with stationary and moving objects. Psychol Rev 1995; 102: 627-51.

[21] Lenoir M, Musch E, Janssens M, Thiery E, Uyttenhove, J. Intercepting moving objects during self-motion. J Motor Behav 1999; 31: 55-67.

[22] Chardenon A, Montagne G, Buekers MJ, Lauent M. The visual control of ball interception during human locomotion. Neurosci Lett 2002; 334: 13-6.

[23] Bastin J, Craig C, Montagne G. Prospective strategies underlie the control of interceptive actions. Hum Mov Sci 2006; 25: 718-32.

[24] Bastin J, Montagne G. The perceptual support of goal-directed displacement is context-dependent. Neurosci Lett 2005; 376: 1216.

[25] Chohan A, Verheul MHG, Savelsbergh GJP, Van Kampen PM, Wind M. Children's use of the bearing angle in interceptive actions. J Motor Behav 2008; 40: 18-28.

[26] Bastin J, Calvin S, Montagne G. Muscular proprioception contributes to the control of interceptive action. J Exp Psychol Hum Percept Perform 2006; 32: 964-72.

[27] Bootsma RJ, Craig CM. Global and local contributions to the optical specification of time to contact: Observer sensitivity to composite tau. Perception 2002; 31: 901-24.

[28] Chardenon A, Montagne G, Laurent M, Bootsma R. The perceptual control of goal-directed locomotion: a common control architecture for interception and navigation. Exp Brain Res 2004; 158: 100-8.

[29] Li L, Warren WH. Perception of heading during rotation: Sufficiency of dense motion parallax and reference objects. Vision Res 2000; 40: 3873-94.

[30] Royden CS, Crowell JA, Banks MS. Estimating heading during eye movements. Vision Res 1994; 34: 3197-214. 